\title{
Diagnóstico y evaluación del uso y aprovechamiento del agua en el Distrito de Riego 05-Delicias, Chihuahua, México
}

\section{Diagnosis and evaluation of water resources use in Irrigation District 05-Delicias, Chihuahua, México}

\begin{abstract}
Octavio Villalobos-Cano 1 [D
Eduardo Santellano-Estrada2 (D)

Esteban Sánchez-Chávez $3 *$ [D,

Pablo Fidel Mancillas-Flores² (D),

Martin Martínez-Salvador 2 (D,

Carlos Raúl Morales-Nieto 2 (D)

Mario Edgar Esparza-Vela2 [D]

${ }^{1}$ Programa de Doctorado en Re-

cursos Naturales de la Facultad de

Zootecnia y Ecología de la Universi-

dad Autónoma de Chihuahua. Km1

Periférico R. Almada, CP. 31453.

Chihuahua, México.

${ }^{2}$ Facultad de Zootecnia y Ecología

de la Universidad Autónoma de Chi-

huahua. Km 1 Periférico R. Almada,

CP. 31453. Chihuahua, México.

${ }^{3}$ Centro de Investigación en Ali-

mentación y Desarrollo A.C. Unidad

Delicias. Avenida cuarta sur, CP.

3828, colonia Pablo Gómez. Delicias,

Chihuahua, México.
\end{abstract}

*Autor de correspondencia:

esteban@ciad.mx

\section{Artículo científico}

Recibido: 20 de marzo de 2019

Aceptado: 10 de septiembre de 2019

Como citar: Villalobos-Cano O Santellano-Estrada E, SánchezChávez E, Mancillas-Flores PF, Martínez-Salvador M, MoralesNieto CR, Esparza-Vela ME (2020) Diagnóstico y evaluación del uso y aprovechamiento del agua en el Distrito de Riego 05-Delicias, Chihuahua, México. Ecosistemas y Recursos Agropecuarios 7(1): e2195. DOI: 10.19136/era.a7n1.2195
RESUMEN. Se abordó la problemática del recurso hídrico en el Distrito de Riego 05-Delicias. El objetivo fue analizar el uso del recurso hídrico mediante indicadores, que permitan plantear alternativas de solución. Se analizaron 21 ciclos agrícolas, por medio de las variables: superficie, volumen, ingreso y sus interacciones, los cuales se evaluaron con estadística descriptiva, análisis de componentes principales, análisis clúster y multivariado de la varianza. Se encontró que la producción presentó variación en el tiempo, incrementándose la superficie y el volumen de cultivos perenes, lo que ocasionó variabilidad en subciclos. Los indicadores de eficiencia productiva y económica indican tres grupos de cultivos, de mayor eficiencia económica, mayor eficiencia productiva, y el último con mayor superficie sembrada y consumo de agua que incluye el cultivo de alfalfa y nogal, los cuales tienen diferencias significativas entre sí y cultivos. Lo que indica diferentes alternativas productivas, que difieren en resultados en impacto ambiental, como la cebolla con la mayor eficiencia productiva y económica. Los indicadores muestran avance en eficiencia del recurso hídrico, pero se recomienda implementar programas que incentiven el uso de riego tecnificado, principalmente en los cultivos de mayor superficie y disminuir de forma paulatina la asignación de volúmenes de agua, para mejorar la eficiencia, donde los cultivos y consumos dependan del volumen asignado.

Palabras clave: Caracterización, recurso hídrico, eficiencia económica, productividad, impacto ambiental.

ABSTRACT. The problem of the resource in the Irrigation District 05-Delicias was addressed. The objective was to analyze the use of this resource, through indicators, which allow for alternative solutions. 21 agricultural cycles were analyzed, using the variables: surface, volume, income and their interactions, which were evaluated with descriptive statistics, principal component analysis, cluster and multivariate analysis of variance. It was found that production showed variation over time, increasing the area and volume of perennial crops, which caused variability in subcycles. The indicators of productive and economic efficiency indicate three groups of crops, of greater economic efficiency, another with greater productive efficiency, and the last one with greater planted area and water consumption that includes alfalfa and pecan cultivation, which have significant differences between groups and crops. Which indicates different productive alternatives, which differ in results in environmental impact, such as the onion that had the highest productive and economic efficiency. The indicators show progress in water resource efficiency, but it is recommended to implement programs that encourage the use of technified irrigation, mainly in larger area crops and gradually reduce the allocation of water volumes, to improve efficiency, where crops and consumptions depend on the assigned volume.

Key words: Characterization, water resource, economic efficiency, productivity, environmental impact. 


\section{INTRODUCCIÓN}

La variabilidad de la producción ocurre por diferentes componentes y formas que se llevan a cabo en un sistema productivo. Para Amaro-Rosales y De Gortari-Rabiela (2016) un sistema productivo está determinado por la participación de los diferentes actores y las relaciones que ocurren al interior del mismo; por lo que en la producción agrícola es de suma importancia identificar de forma clara estas características. Los sistemas de producción se caracterizan por su alta complejidad y su relación intrínseca con el medio ambiente (Avilez et al. 2010). Una caracterización de la producción y de la productividad, identificando los elementos que intervienen en el sistema, permite valorar las particularidades importantes en el uso de los recursos para mejorar su eficiencia productiva (Tapella y Rodríguez-Bilella 2014). Al respecto, Sánchez et al. (2017) señalan que identificar las características es la base de la gestión estratégica para el desarrollo sostenido, ya que el conjunto de recursos no es igual para todos los productores. También son diferentes las competencias y capacidades que conducen a diferentes resultados con base en el uso de los recursos, por lo que se debe identificar las características que produzcan mayor rentabilidad económica, social y ambiental.

Las diferencias en la productividad dependen de las características distintivas de la producción, del uso de los factores productivos y de la combinación de los mismos, por lo que una caracterización permite describir los principales rasgos distintivos y las múltiples interrelaciones (Hofman et al. 2017). Al respecto, Aravena y Hofman (2013) mencionan que el decremento de la productividad es un reflejo del uso no adecuado de los recursos, lo que repercute en deterioro ambiental. El uso eficiente de los recursos es fundamental para garantizar la producción alimentaria y mantener el trabajo de las familias vinculadas con el sector agrícola, por lo que es urgente incrementar la eficiencia productiva, entendiendo eficiencia o productividad como la relación entre la producción de un cultivo por unidad de insumo (SalazarMoreno et al. 2014). Se deben buscar estrategias que permitan elevar los índices de productividad para lograr desarrollo económico y cumplir con el objetivo de proveer de alimentos (CONAGUA 2014).

El conocimiento de la problemática existente proporciona el soporte para tomar decisiones acertadas y realizar cambios estructurales, buscando una solución económicamente eficiente, socialmente equitativa y ambientalmente sustentable, que promueva el desarrollo en mejora de la calidad de vida de la población (Palacio-Velez y Mejia-Saez 2011). Aumentar la producción y productividad agrícola es el mayor reto para mejorar la seguridad alimenticia y reducir la pobreza (Olvera-Salgado et al. 2014). La problemática del DR-05, sólo se ha analizado bajo perspectivas de variables técnicas-productivas, principalmente del uso y manejo del recurso agua (Brenes-Gámez et al. 2000, Ortega-Gaucin 2009, Olvera-Salgado et al. 2014, Ríos et al. 2017). Pero no hay estudios que caractericen la variabilidad de la producción y productividad dentro del distrito de riego DR-05, con énfasis en la preservación y uso sustentable de los recursos naturales para el beneficio social. Por lo anterior, el objetivo del presente trabajo fue caracterizar la producción y productividad en el DR-05, para establecer las bases de un uso adecuado de los insumos productivos que incida en beneficios económicos, sociales y ambientales en la región.

\section{MATERIALES Y MÉTODOS}

\section{Descripción del área y población de estudio}

El Distrito de Riego 05 (DR-05) está ubicado en la región Centro Sur del Estado de Chihuahua, México, en la cuenca hidrológica del Río Bravo, a $28^{\circ} 11^{\prime}$ LN y $105^{\circ} 28^{\prime} \mathrm{LO}$, con altitud promedio de $1165 \mathrm{msnm}$ (Figura 1), tiene una superficie de 73 002 ha de riego y un padrón de 8113 productores, $43 \%$ ejidatarios y $57 \%$ pequeños productores, que se distribuyen en 55 secciones de riego. Se agrupan en 10 asociaciones civiles o módulos de riego, que se asocian en dos sociedades de responsabilidad limitada (SRL). La población de estudio fueron los 8113 productores que conforman el padrón de usuarios y representan la totalidad de la superficie de riego del 


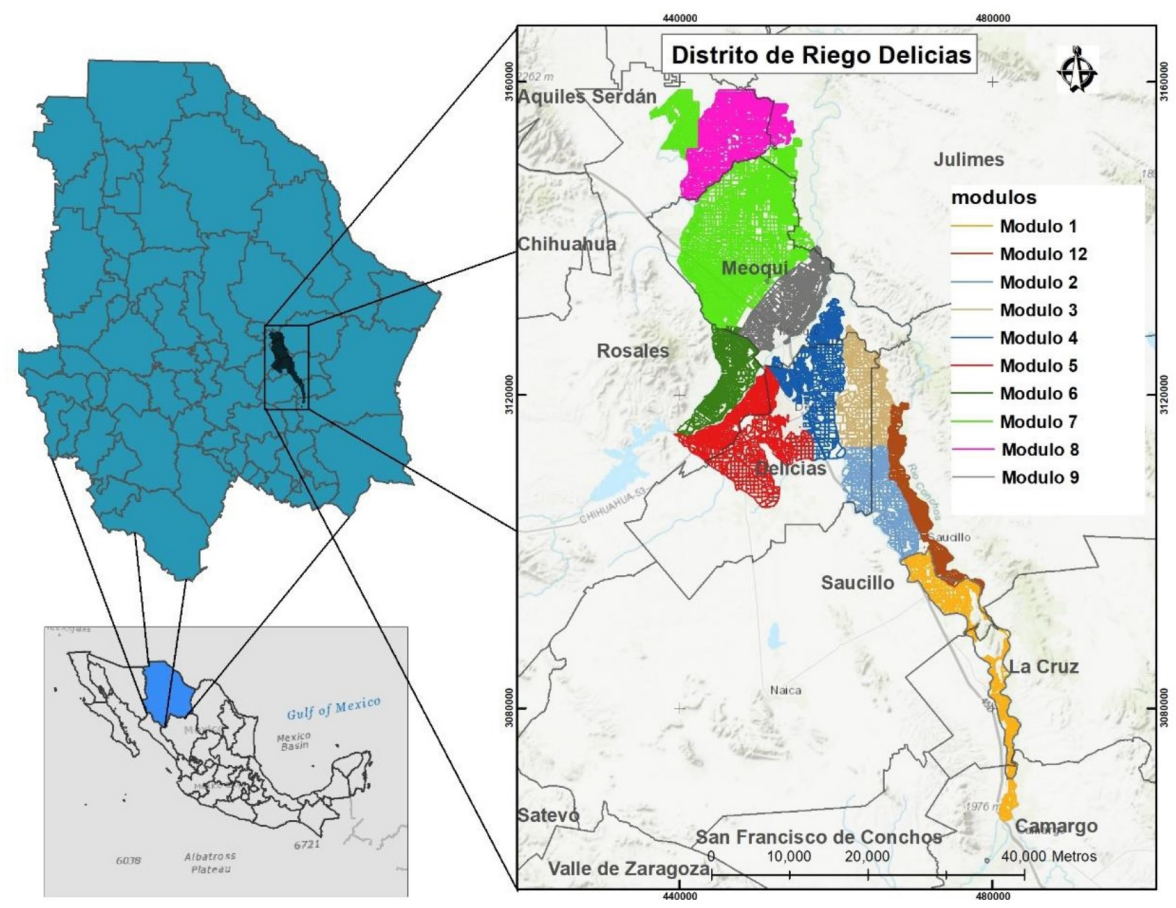

Figura 1. Localización del Distrito de Riego 05-Delicias (DR-05) en el Estado de Chihuahua, México.

DR-05.

\section{Tipo de investigación}

Se realizó una investigación de tipo exploratoria, descriptiva y conclusiva. Comprendió un tema no abordado con anterioridad en el DR-05, sus resultados constituyen una visión aproximada del objeto de investigación. Determina propiedades, características y perfiles importantes del proceso analizado. Su objetivo es ayudar a la toma de decisiones manifestando conclusiones de los procesos mediante el análisis de la información.

\section{Acceso a la información y elección de los cultivos de estudio}

La revisión de información estadística de la producción agrícola fue de tipo documental mediante el acceso a diferentes bases de datos de la SEMARNAT del 2010, 2011, 2012, 2013, 2014, 2015, 2016 y 2017, que se corroboró y complementó con entrevistas que se realizaron de agosto de 2014 a octubre de 2018 a las autoridades de la CONAGUA, Jefe de Distrito de Riego, Jefe de Operación, gerentes de las asociaciones civiles de usuarios y de las $S R L$ que conforman el DR-05. Para generar la información se consideraron todos los cultivos registrados en los últimos 21 ciclos agrícolas, tomando como base los registros de información de asociaciones que comprenden el DR-05.

\section{Tipo de variables}

De los diferentes ciclos agrícolas y cultivos considerados en el estudio, se obtuvieron las variables superficie sembrada (SUPSEM, ha), producción (PROD, t), valor de la producción (VALOR, $\$)$, volumen utilizado de agua $\left(\mathrm{VOL}, \mathrm{dam}^{3}\right)$, ingresos obtenidos por unidad de volumen de agua (VALVOL, $\$$ dam $^{-3}$ ), valor de la producción por unidad de superficie (VALORHA, $\$ \mathrm{ha}^{-1}$ ), volumen de agua utilizado por unidad de superficie (VOLHA, dam ${ }^{3} \mathrm{ha}^{-1}$ ) y volumen utilizado para obtener una unidad producto (VOLTON, dam $\left.{ }^{3} \mathrm{t}^{-1}\right)$.

\section{Análisis estadístico}

Se analizaron 21 ciclos agrícolas, con estadística descriptiva de acuerdo con la metodología 
de Hernández-Sampieri et al. (2010). Para luego realizar un análisis de Componentes Principales (ACP), Conglomerados (AC) y análisis multivariado de la varianza (MANOVA), con los procedimientos PRINCOMP, CLUSTER y MANOVA del paquete $S A{ }^{\circledR}$. El análisis clúster se realizó con el método Ward, determinando los integrantes del clúster por medio de la suma de las desviaciones al cuadrado de las observaciones con respecto a la media, expresado en un coeficiente de determinación parcial (Álvarez y Massa 2015). Se consideraron para el análisis de estadística descriptiva el periodo de 19962017, para analizar comparativamente mediante el ACP, AC y MANOVA se seleccionaron dos ciclos agrícolas representativos, no atípicos, 2009-2010 (T1) y 2016-2017 (T2) y otro promedio de los últimos seis

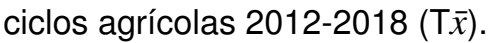

\section{RESULTADOS}

\section{Caracterización de la producción por ciclo y cul- tivo en el DR-05}

La caracterización determino los elementos del proceso productivo para valorar su eficiencia. El comportamiento de la producción en el DR-05 en los últimos 21 ciclos agrícolas (1996 a 2017) mostró variación a lo largo del tiempo con incremento de la superficie y volumen utilizado por cultivos perenes (PER) y la disminución de otros cultivos. A nivel de ciclo agrícola la superficie sembrada presentó un coeficiente de variación (CV) del $38.36 \%$, con valor máximo y mínimo de 78525 y 19454 ha, respectivamente. A nivel subciclo, primavera-verano $(P V)$ y PER fueron los que presentaron menor variación, en superficie sembrada con respecto al tiempo, con CV de 29.77 y $46.29 \%$ respectivamente; mientras que los de mayor variación fueron ciclos otoño-verano (2C) y otoño-invierno (OI, Figura 2). Los cultivos perenes aumentaron de forma gradual con el tiempo, representando en el ciclo agrícola 2016-2017 el 63.23\% de la superficie total sembrada en el DR-05. La variabilidad en la superficie sembrada de cultivos perenes se debe al incremento gradual positivo con el tiempo, teniendo un $\frac{d x}{d y}$ de 1451.90 ha por año, con $\mathrm{R}^{2}$ del $72.55 \%$, en tanto que los cultivos PV tuvieron una pendiente de 153.72 ha, con explicación del factor tiempo del $2.34 \%$ de la superficie sembrada (Figura 3).

La variabilidad por cultivo, indica que el trigo, tomate y soya son los que tienen el mayor CV con valores de $276.09,263.22$ y $242.51 \%$, respectivamente; al grado que estos cultivos no tienen registros en los últimos cinco ciclos. Por otra parte, los cultivos que menor variabilidad, en la superficie sembrada, presentaron fueron el chile, maíz, cebolla, nogal, alfalfa y cacahuate, con CV del 26.52, 44.26. 45.05, $45.39,48.99$ y $55.26 \%$ respectivamente (Figura 4 ). Con registros de producción en todos los ciclos, siendo los cultivos más importantes en el DR-05 y referentes a nivel nacional, en base a superficie sembrada y valor de la producción. Mientras que los cultivos alfalfa y nogal mostraron mayor crecimiento en superficie, con tasa del 278 y $240 \%$.

\section{Análisis multivariado de la producción y produc- tividad en el DR-05}

El análisis de correlación entre pares de variables indica asociación lineal positiva, la cual fue estadísticamente significativa en la mayoría de los casos. Lo que sugiere realizar el análisis de los datos por medio de Componentes Principales en los periodos de tiempo 2009-2010 (T1), 2016-2017 (T2), y 2012-2018 (T. $\bar{x})$. La Tabla 1 muestra los estadísticos descriptivos, en la que se observa disminución en el VOLHA utilizado, pero se observa un incremento en VOLTON, lo que indica disminución en la productividad del agua (T1 vs T2). Las mayores variaciones mostradas son consecuencia de las oscilaciones en la superficie y el volumen utilizado, con tendencia a incrementar en ambos parámetros, 1116.3 ha y 8 714.9 dam $^{3}$ por ciclo agrícola, con coeficiente de determinación de 0.91 (Figura 5).

\section{Análisis de componentes principales}

Los cultivos establecidos en los ciclos T1, T2 y $\mathrm{T} \bar{x}$ fueron 18, 9 y 12 respectivamente, presentando dispersión estadística en sus variables, pero con correlación significativa alta. Donde cada eigenvalor indica la variación que es explicada por su com- 


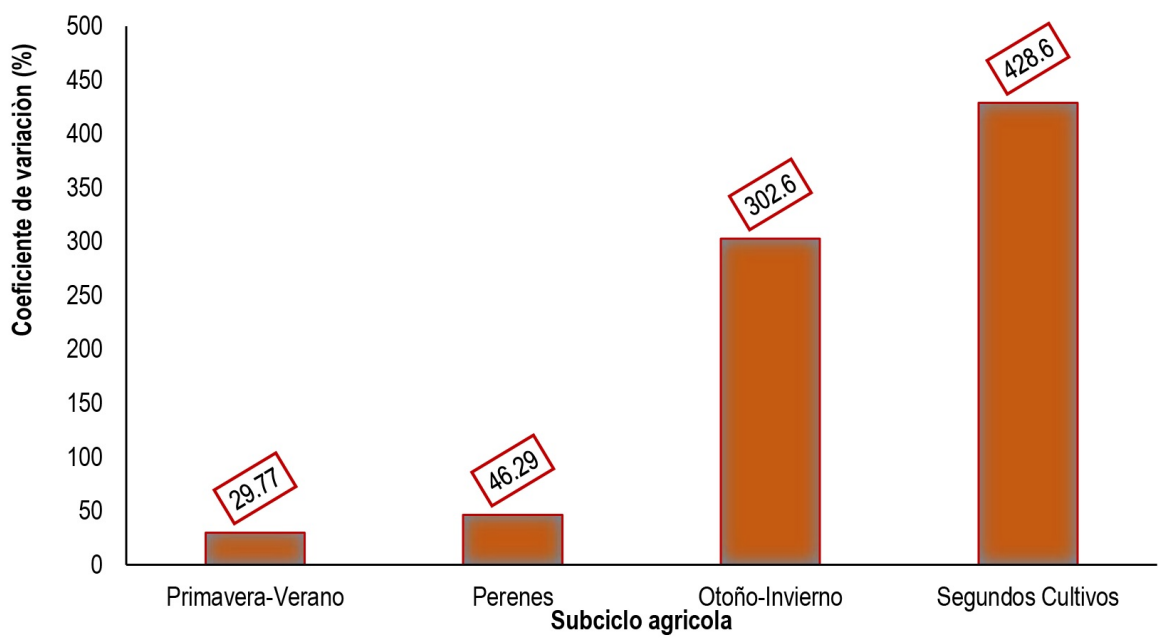

Figura 2. Variabilidad por subciclo en el Distrito de Riego 05-Delicias.

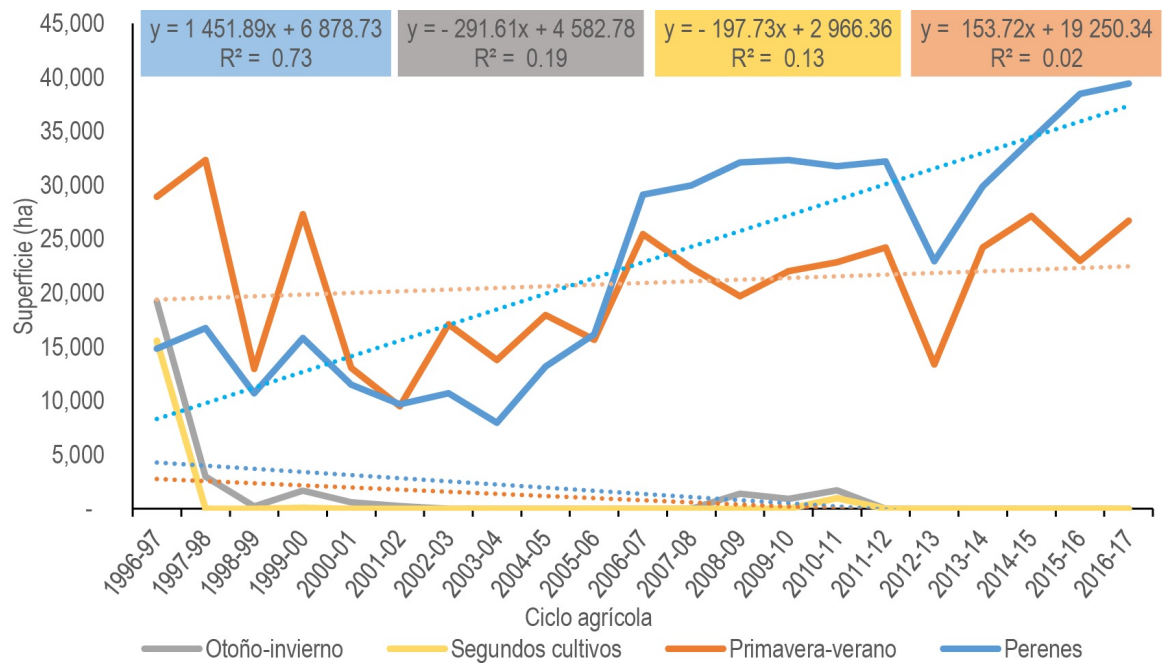

Figura 3. Variación y tendencia por subciclo agrícola en el Distrito de Riego 05-Delicias, Chihuahua.

ponente principal (CP) derivado. La Tabla 2 presenta las variables, $\mathrm{CP}$, eigenvectores, coeficientes de correlación, eigenvalores y varianza explicativa de cada CP, donde se observa en T1 que el CP1 y CP2 explican el $77.99 \%$ de la variación total, mientras en T2 el 78.66 y en $T \bar{x}$ el $81.30 \%$ de la variabilidad.

En T1, T2 y T $\bar{x}$ el CP1 explica el 51 y $54 \%$ de la varianza total, presentando mayor influencia por indicadores de producción (SUPSEM, PROD, VOL, VALOR y VOLHA) con coeficientes muy similares para las cinco variables, mientras que el CP2 mostró mayor poder explicativo con fuerte asociación con indicadores de productividad económica (variables VALVOL y VALHA). De acuerdo a lo anterior, y con base al CP1 se encontró que los cultivos más importantes, por sus indicadores de producción en T1 fueron: Alfalfa, chile, nogal y cebolla, mientras que en T2 y $T \bar{x}$ fueron: Alfalfa, nogal y chile, pero tomando como base el CP2 los cultivos de mayor eficiencia productiva en T1 fueron: cebolla, tomate, chile y sandia, y en T2 fueron cebolla, chile y sandia, en T $\bar{x}$ fueron varios, cebolla, chile, nogal y sandia (Figura 6). Es importante mencionar que en alfalfa, nogal, chile, cebolla y sandia se ha llevado a cabo un proceso 


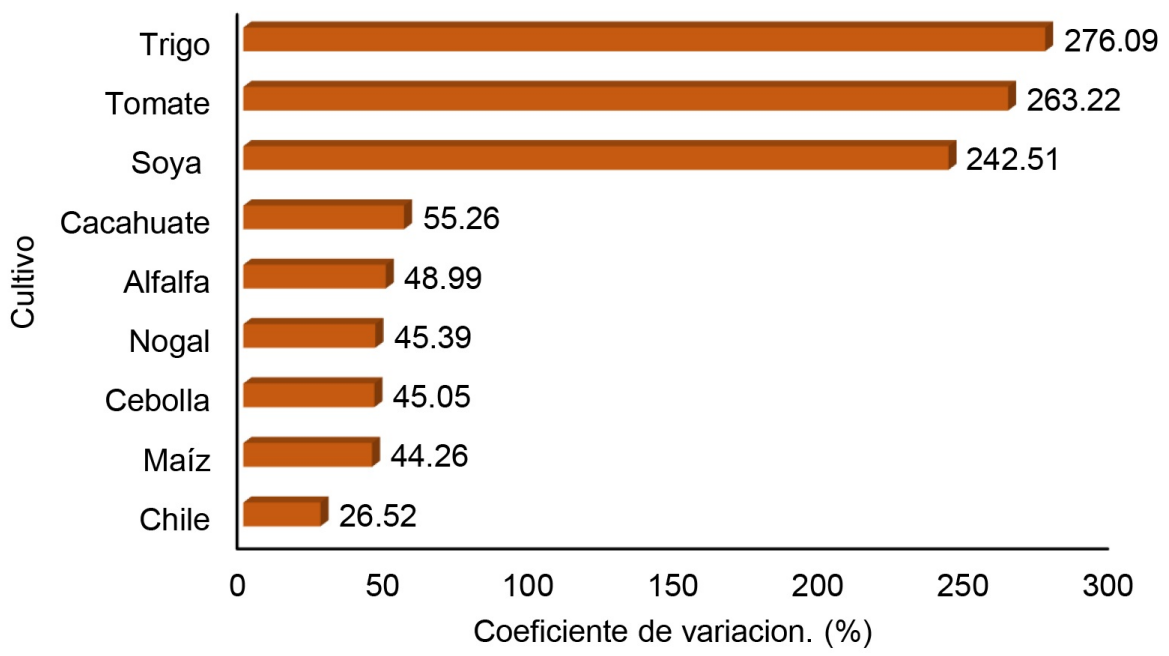

Figura 4. Coeficiente de variación (CV) en porcentaje de los cultivos, ciclos 1996-2017, en el Distrito de Riego 05-Delicias, Chihuahua.

Tabla 1. Estadísticos descriptivos de las variables del Distrito de Riego 05-Delicias, Chihuahua.

\begin{tabular}{lccccccc}
\hline Variable & Ciclo & Media & Dev std & Coef var & Suma & Mínimo & Máximo \\
\hline SUPSEM & $2009-2010$ & 3071 & 6088 & 198.24 & 55286 & 11 & 26133 \\
PROD & $2009-2010$ & 63676 & 128726 & 202.16 & 1146164 & 143 & 522660 \\
VALOR & $2009-2010$ & 107999805 & 183569876 & 169.97 & 1943996493 & 115500 & 705591000 \\
VOL & $2009-2010$ & 46020 & 108054 & 234.80 & 828359 & 185 & 465577 \\
VALORVOL & $2009-2010$ & 2851 & 2427 & 85.13 & 51318 & 597 & 10384 \\
VALORHA & $2009-2010$ & 34443 & 31405 & 91.18 & 619966 & 8250 & 120000 \\
VOLTON & $2009-2010$ & 2.15 & 3.12 & 145.14 & 38.71 & 0.19 & 9.49 \\
VOLHA & $2009-2010$ & 12.15 & 3.12 & 25.67 & 218.78 & 5.23 & 17.82 \\
SUPSEM & $2016-2017$ & 7347 & 8409 & 114.45 & 66124 & 1478 & 28649 \\
PROD & $2016-2017$ & 316083 & 587392 & 185.83 & 2844744 & 4433 & 1862203 \\
VALOR & $2016-2017$ & 658930825 & 483778320 & 73.42 & 5930377422 & 83780487 & 1396652400 \\
VOL & $2016-2017$ & 100905 & 138232 & 136.99 & 908147 & 12693 & 455246 \\
VALORVOL & $2016-2017$ & 10233 & 7042 & 68.82 & 92100 & 2721 & 21387 \\
VALORHA & $2016-2017$ & 113161 & 69401 & 61.33 & 1018450 & 37500 & 210000 \\
VOLTON & $2016-2017$ & 2.24 & 3.78 & 168.83 & 20.13 & 0.13 & 11.55 \\
VOLHA & $2016-2017$ & 11.86 & 2.65 & 22.37 & 106.78 & 8.59 & 15.89 \\
SUPSEM & $2012-2018$ & 4813 & 6924 & 143.86 & 57755 & 27 & 25080 \\
PROD & $2012-2018$ & 158097 & 370398 & 234.29 & 1897160 & 328 & 1320895 \\
VALOR & $2012-2018$ & 339750852 & 369426566 & 108.73 & 4077010218 & 590400 & 1016820236 \\
VOL & $2012-2018$ & 67746 & 113367 & 167.34 & 812957 & 285 & 407947 \\
VALORVOL & $2012-2018$ & 6270 & 5282 & 84.24 & 75234 & 845 & 16112 \\
VALORHA & $2012-2018$ & 74547 & 61205 & 82.10 & 894566 & 7641 & 175139 \\
VOLTON & $2012-2018$ & 1.81 & 2.78 & 153.22 & 21.77 & 0.18 & 9.88 \\
VOLHA & $2012-2018$ & 11.77 & 2.23 & 18.95 & 141.26 & 9.04 & 16.27
\end{tabular}

SUPSEM = Superficie sembrada; $P R O D=$ Producción; VALOR $=$ Valor de la producción, $\mathrm{VOL}=$ Volumen de agua utilizado; VALORVOL = Pesos obtenidos por cada unidad de agua; VALORHA $=$ Pesos obtenidos por hectárea, VOLTON $=$ Volumen por tonelada; VOLHA = Volumen utilizado por hectárea; Coef var = Coeficiente de variación.

de especialización de forma natural, no inducida, llegando a posicionarse en los últimos 10 ciclos agrícolas en los primeros puestos a nivel nacional, en el caso de los cultivos con mayores consumos de agua por unidad como la alfalfa y el nogal, se ha incrementado la superficie establecida de estos cultivos poniendo en riesgo la estabilidad ambiental por el aumento en los volúmenes de agua necesarios, repercutiendo en la estabilidad económica y social, por lo que se deben considerar que los incrementos de superficies de estos cultivos condicionan la disponibilidad del recurso, y deben tomarse en cuenta estos 


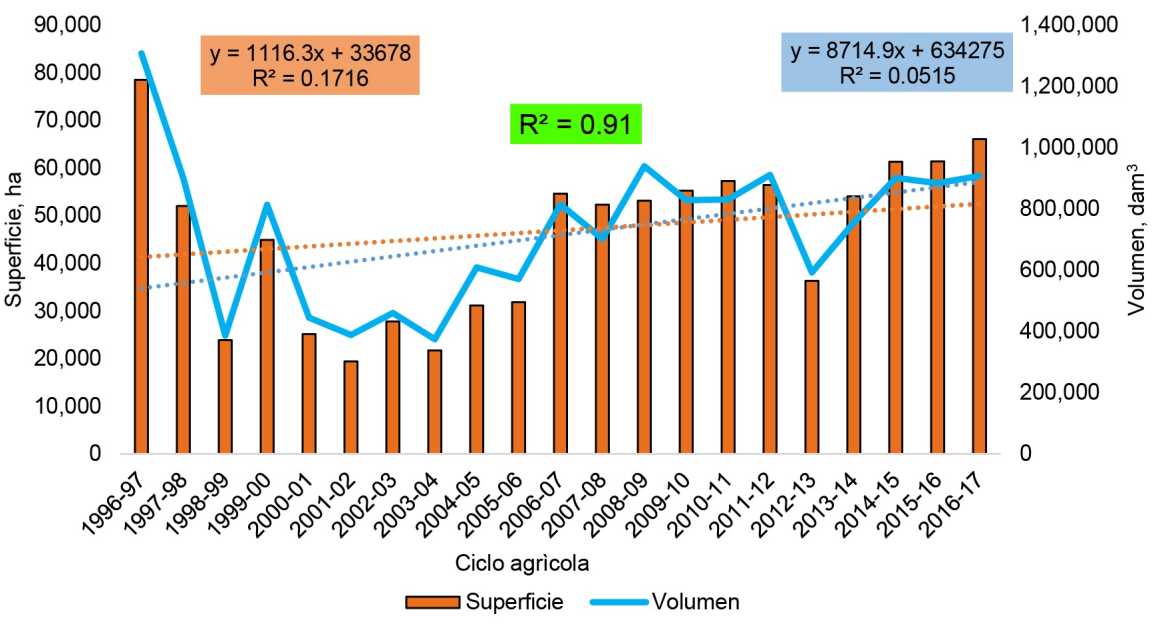

Figura 5. Superficie sembrada y volumen utilizado en el Distrito de Riego 05-Delicias, 1996-2018.

Tabla 2. Correlación, varianza explicativa por componente principal y variable.

\begin{tabular}{|c|c|c|c|c|}
\hline Ciclo/Variable & $\begin{array}{l}\text { CP1 } \\
\text { Eigenvector }\end{array}$ & $\begin{array}{l}\text { CP1 } \\
\text { Correlación }\end{array}$ & $\begin{array}{l}\text { CP2 } \\
\text { Eigenvector }\end{array}$ & $\begin{array}{l}\text { CP2 } \\
\text { Correlación }\end{array}$ \\
\hline \multicolumn{5}{|l|}{$2009-2010$} \\
\hline SUPSEM & 0.4738 & 0.9652 & -0.1303 & -0.1883 \\
\hline PROD & 0.4712 & 0.9599 & -0.0145 & -0.0210 \\
\hline VALOR & 0.4732 & 0.9640 & 0.0602 & 0.0870 \\
\hline VOL & 0.4734 & 0.9644 & -0.1339 & -0.1935 \\
\hline VALORVOL & 0.0405 & 0.0826 & 0.6750 & 0.9756 \\
\hline VALORHA & 0.1005 & 0.2047 & 0.6613 & 0.9558 \\
\hline VOLTON & -0.0555 & -0.1132 & -0.2611 & -0.3773 \\
\hline VOLHA & 0.3011 & 0.6135 & -0.0161 & -0.0233 \\
\hline Eigrenvalor & 4.1502 & & 2.0891 & \\
\hline Varianza explicativa, \% & 51.8775 & & 26.1133 & \\
\hline $\begin{array}{l}\text { Varianza acumulada, \% } \\
2016-2017\end{array}$ & 51.8775 & & 77.9907 & \\
\hline SUPSEM & 0.4667 & 0.9729 & 0.1061 & 0.1481 \\
\hline PROD & 0.4294 & 0.8951 & 0.1468 & 0.2049 \\
\hline VALOR & 0.3213 & 0.6698 & 0.4256 & 0.5938 \\
\hline VOL & 0.4690 & 0.9778 & 0.0987 & 0.1377 \\
\hline VALORVOL & -0.2746 & -0.5726 & 0.5788 & 0.8076 \\
\hline VALORHA & -0.2192 & -0.4569 & 0.6241 & 0.8708 \\
\hline VOLTON & 0.0270 & 0.0563 & -0.2273 & -0.3171 \\
\hline VOLHA & 0.3879 & 0.8087 & 0.0162 & 0.0226 \\
\hline Eigrenvalor & 4.3462 & & 1.9469 & \\
\hline Varianza explicativa, \% & 54.3278 & & 24.3361 & \\
\hline $\begin{array}{l}\text { Varianza acumulada, \% } \\
2012-2018\end{array}$ & 54.3278 & & 78.6639 & \\
\hline SUPSEM & 0.4638 & 0.9631 & -0.1404 & -0.2078 \\
\hline PROD & 0.4249 & 0.8823 & -0.1524 & -0.2257 \\
\hline VALOR & 0.4340 & 0.9014 & 0.2326 & 0.3444 \\
\hline VOL & 0.4648 & 0.9652 & -0.1503 & -0.2225 \\
\hline VALORVOL & 0.0157 & 0.0326 & 0.6573 & 0.9731 \\
\hline VALORHA & 0.0781 & 0.1623 & 0.6644 & 0.9836 \\
\hline VOLTON & 0.0708 & 0.1470 & 0.0066 & 0.0098 \\
\hline VOLHA & 0.4343 & 0.9019 & 0.0830 & 0.1229 \\
\hline Eigrenvalor & 4.3126 & & 2.1918 & \\
\hline Varianza explicativa, \% & 53.91 & & 27.40 & \\
\hline Varianza acumulada, \% & 53.91 & & 81.30 & \\
\hline
\end{tabular}


Ciclo 2009-2010

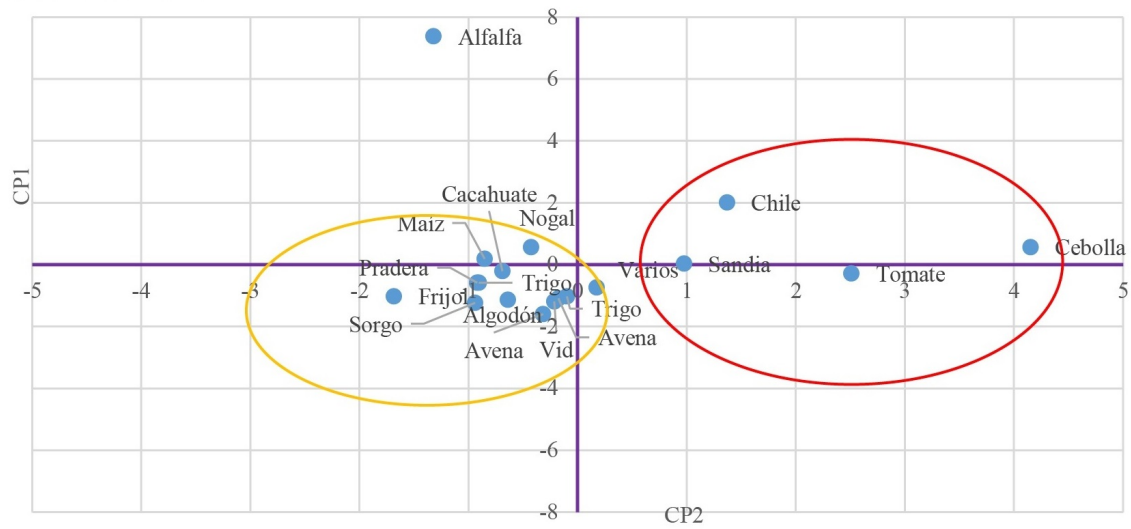

Ciclo 2016-2017

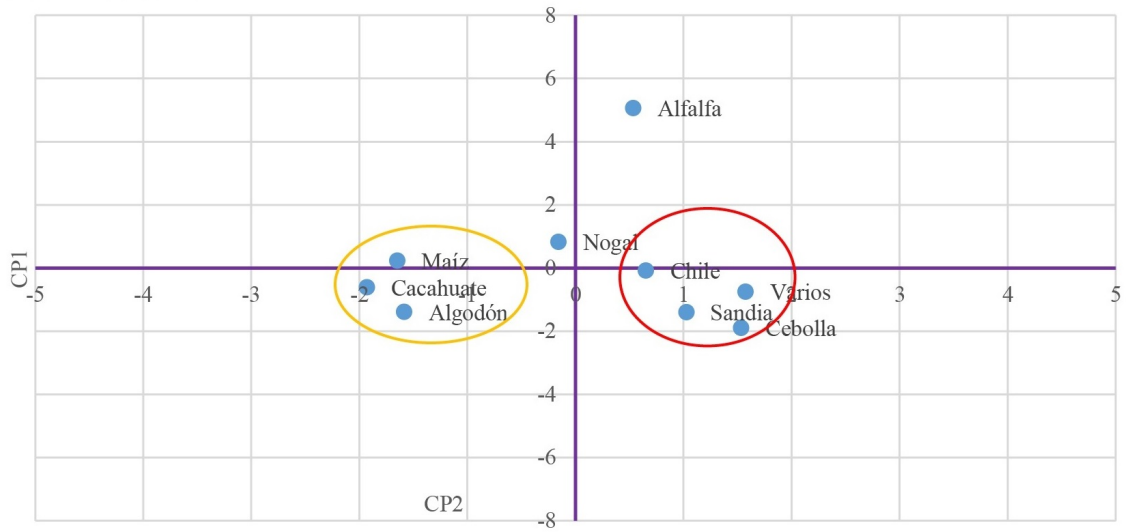

Ciclos 2012-2018

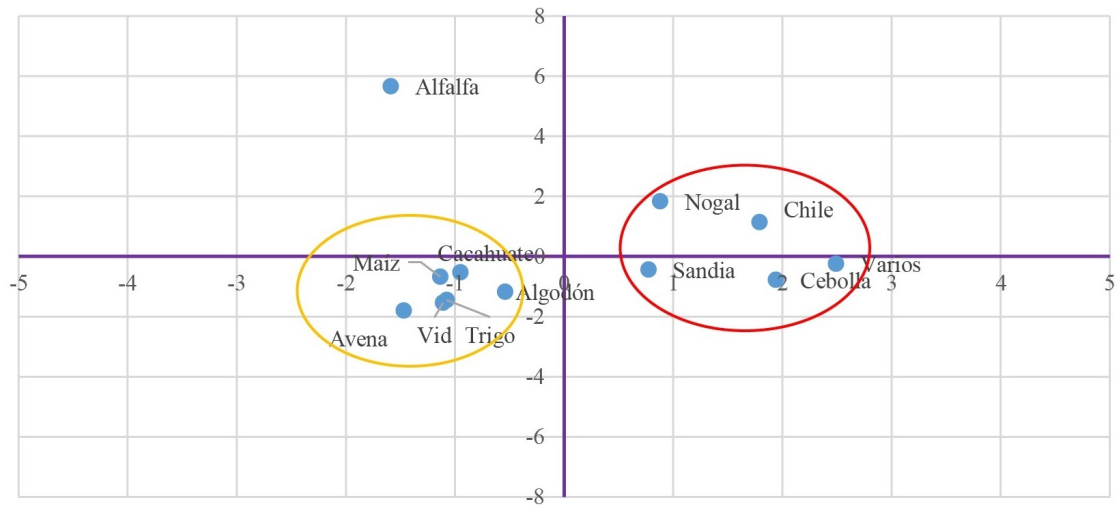

Figura 6. Importancia de los cultivos en el Distrito de Riego 05-Delicias, Chihuahua, en base a componentes principales 1 y 2 (CP1 y CP2).

factores para realizar cualquier programación de cultivos y volumen requerido para los mismos y para el buen funcionamiento del DR-05.

\section{Análisis de conglomerados}

El ACP indica una tendencia de integración de los cultivos en grupos, como se observa en la Figura 7 , en base a sus características productivas y de eficiencia económica principalmente, por lo cual, para 


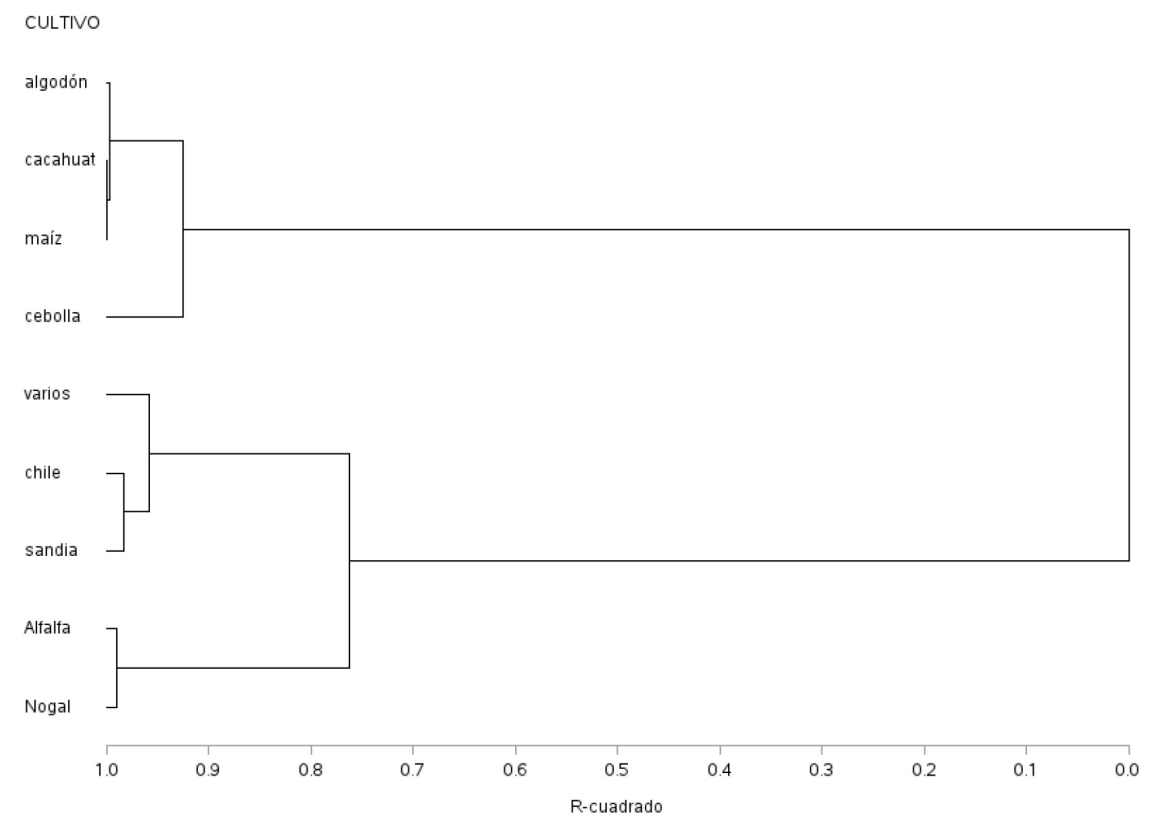

Ciclo 2012-2018

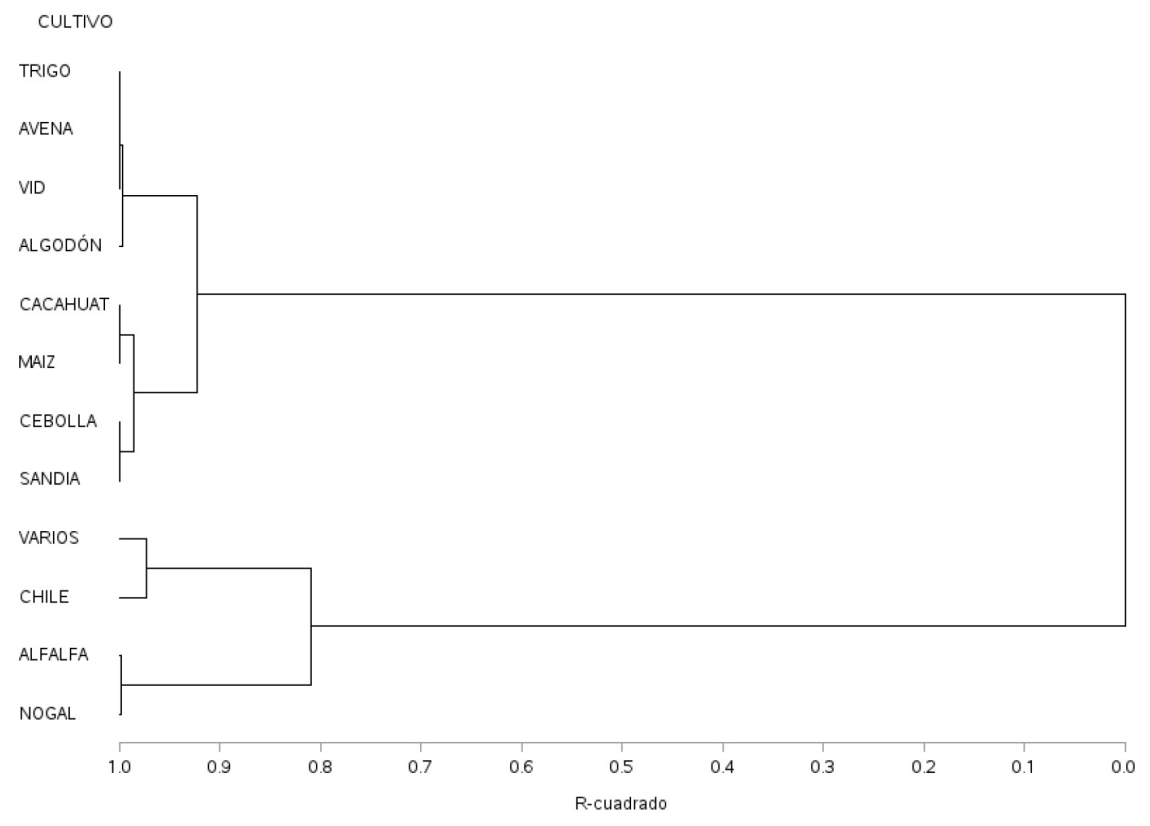

Figura 7. Grupos de cultivos generados mediante análisis clúster.

validar esto, se realizó un análisis de conglomerados, aun cuando en los ciclos analizados mediante ACP, T1, T2 y T $\bar{x}$ hay diferentes números de cultivos y condiciones, con similares tendencias de agrupación en base a sus características productivas y de productividad física y económica, por lo que se decidió tomar como base para el AC a T2 como un ciclo agrícola típico reciente y $T \bar{x}$, que representa el promedio los últimos seis ciclos agrícolas, y que mediante el ACP presentaron ambos la tendencia de 
integrarse en dos grupos plenamente diferenciados, en base a máxima heterogeneidad entre estos y homogeneidad al interior de los mismos, mediante los valores de $R^{2}$, donde en un primer grupo (G1EE) se integraron los cultivos que se han posicionado como referentes a nivel nacional, que han tenido un proceso de especialización en el DR-05, con mayor eficiencia económica y productiva, pero con mayores consumos de agua y variabilidad en los factores económicos que inciden sobre el mercado, principalmente precio del producto. Por otra parte, el segundo grupo (G2PROD) está compuesto por cultivos de menor eficiencia económica, pero con menor consumo de agua y variabilidad con respecto al precio, representando un menor ingreso esperado comparativamente, pero con mayor certeza en el mismo. Lo que representa dos alternativas productivas en base a la aversión al riesgo, por ende, del tipo de productor, de su capacidad económica y disponibilidad de recursos.

El cultivo de cebolla se integró en el grupo dos, pero presenta características representativas de ambos grupos, del primero eficiencia económicaproductiva y variabilidad en el precio, y del segundo grupo los menores consumos de agua, siendo la cebolla el cultivo que presentó el mejor desempeño de eficiencia del uso del agua, reduciendo sus consumos por unidad de superficie en un 55\%, pasando en un periodo de 21 ciclos agrícolas (1996-2017), de consumos de 21.75 a 9.82 dam $^{3}$ ha $^{-1}$. Además, el cultivo de chile mejoró su eficiencia reduciendo su consumo en un $44 \%$ en el mismo periodo, estas mejoras en la eficiencia del uso del agua son deben a la implementación de nuevas tecnologías como el riego subsuperficial, pasando de valores de 25.35 a 14.28 $\mathrm{dam}^{3} \mathrm{ha}^{-1}$.

Los cultivos de alfalfa y nogal se integraron en un tercer grupo (G3ALFNOG) con características similares entre ellos, con las mayores superficies establecidas (43.33 y $16.29 \%$ ) y volúmenes utilizados de agua $(50.13$ y $15.75 \%$ ) del total del DR-05, pero con diferencias entre sí, donde el cultivo de alfalfa presenta menor variabilidad del precio y requiere menores inversiones. Con retorno de la inversión gradual de acuerdo a los cortes que se llevan a cabo durante el ciclo agrícola, mientras que el nogal re- quiere mayor inversión, con mayor variabilidad y especulación del precio, pero muestra mayor eficiencia económica por unidad de volumen y de superficie. El cultivo de alfalfa presenta características de los grupos uno (G1EE) y dos (G2PROD), alto consumo de agua como los del G1EE y poca variabilidad del precio y comparativamente menores ingresos por unidad de volumen, con valores en el ciclo agrícola 2016-2017 de 156 US\$ dam ${ }^{-3}$ como el G2PROD.

\section{Análisis multivariado de la varianza}

Para validar las diferencias significativas entre cultivos y grupos, previamente conformados mediante AC se procedió a realizar el análisis multivariado de la varianza (MANOVA) en T $\bar{x}$. La estadística descriptiva básica del análisis multivariado (Tabla 3) mostró respecto a las variables económicas VALVOL ( $\$$ dam $^{3}$ ) y VALHA (\$ ha), que expresan el valor generado en pesos, que los cultivos con mayor eficiencia fueron cebolla (14 298, 144 959), chile (9 $741,150611)$ y varios (12 203, 139000$)$, y los que mostraron menor eficiencia fueron maíz (2 061, 27 $152)$, cacahuate $(2868,31785)$ y alfalfa ( 2463,40 320).

Con respecto a las variables productivas, volumen por hectárea (VOLHA, dam ${ }^{3} \mathrm{ha}^{-1}$ ) y volumen por tonelada (VOLTON, dam ${ }^{3}$ ton $^{-1}$ ), que indican la cantidad del recurso hídrico utilizado por unidad de superficie y por unidad de producto respectivamente, se encontró que la cebolla presentó mejor eficiencia productiva con respecto al volumen de agua utilizada (10.05, 0.19), seguido de algodón y cacahuate. Los de menor eficiencia fueron alfalfa $(16.49,0.35)$ y nogal $(14.16,10.06)$. Mediante el análisis MANOVA se comprobó las diferencias entre grupos (Tabla 4), estableciéndose el G1EE, G2PROD y G3ALFNOG. El G1EE mostró los mejores desempeños de eficiencia económica en términos de VALVOL y VALHA, mientras que el grupo G2PROD tiene la característica de agrupar los cultivos con mejor desempeño productivo del uso del agua en términos de VOLHA, el G3ALFNOG muestra diferencias muy significativas con los demás cultivos en lo referente a SUPSEM y VOL, siendo el grupo que presentó las diferencias más grandes con respecto 
Tabla 3. Estadística descriptiva básica del análisis multivariado de la varianza.

\begin{tabular}{lcccc}
\hline & \multicolumn{2}{c}{ VALVOL } & \multicolumn{2}{c}{ VALHA } \\
& Media + E.E. & D.E. & Media + E.E. & D.E. \\
\hline Alfalfa & $2463.17 \pm 351.09$ & 859.99 & $40319.83 \pm 5541.51$ & 13573.86 \\
Algodón & $4696.00 \pm 485.39$ & 1188.95 & $47379.33 \pm 3464.61$ & 8486.52 \\
Cacahuate & $2867.50 \pm 262.90$ & 643.97 & $31784.50 \pm 1903.12$ & 4661.68 \\
Cebolla & $14298.00 \pm 2290.56$ & 5610.69 & $144959.17 \pm 23672.21$ & 57984.84 \\
Chile & $9740.50 \pm 1495.39$ & 3662.94 & $150610.67 \pm 22931.40$ & 56170.23 \\
Maíz & $2060.67 \pm 348.80$ & 854.39 & $27151.67 \pm 4082.63$ & 10000.36 \\
Nogal & $7278.83 \pm 1082.13$ & 2650.67 & $101792.83 \pm 14842.52$ & 36356.60 \\
Sandia & $8963.67 \pm 2003.91$ & 4908.56 & $106178.33 \pm 18317.98$ & 44869.70 \\
Varios & $12203.00 \pm 3242.84$ & 7943.30 & $139000.00 \pm 33836.37$ & 82881.84 \\
\hline VALVOL $=$ Valor por unidad de volumen agua $\left(\$\right.$ dam $\left.^{-3}\right)$, VALHA $=$ Valor por $_{\text { unidad de superfi- }}$ \\
cie $\left(\$\right.$ ha $\left.^{-1}\right)$, VOLHA $=$ Volumen de agua por unidad de superficie $\left(\right.$ dam $^{3}$ ha $\left.^{-1}\right)$.
\end{tabular}

Tabla 4. Análisis multivariado de grupos de cultivos, valor del estadístico, valor de F y p value, ciclos 2012-2018.

\begin{tabular}{lccc}
\hline \multicolumn{1}{c}{ Análisis } & $\begin{array}{c}\text { Estadístico } \\
\text { Lambda de Wilks }\end{array}$ & Valor de F & Valor de $\mathrm{p}$ \\
\hline MODELO MULTIVARIADO & 0.00004 & 16.490 & $<.0001$ \\
G1EE vs G2PROD & 0.28700 & 11.800 & $<.0001$ \\
G1EE vs G3ALFNOG & 0.03792 & 120.530 & $<.0001$ \\
G2PROD vs G3ALFNOG & 0.03899 & 117.060 & $<.0001$ \\
G1EE+CEBOLLA vs G2PROD & 0.19980 & 19.020 & $<.0001$ \\
ALFALFA vs G1EE & 0.02736 & 168.870 & $<.0001$ \\
ALFALFA vs G2PROD & 0.02437 & 190.190 & $<.0001$ \\
ALFALFA vs NOGAL & 0.02447 & 189.350 & $<.0001$ \\
NOGAL vs G1EE & 0.03695 & 123.800 & $<.0001$ \\
NOGAL vs G2PROD & 0.04459 & 101.770 & $<.0001$ \\
\hline G1EE = Grupo uno eficiencia económica, G2PROD = Grupo dos eficiencia produc- \\
tiva, G3ALFNOG = Grupo tres alfalfa y nogal. & &
\end{tabular}

a los otros dos grupos. Por lo que se procedió a analizar al interior del modelo, mediante comparación de medias mediante Tukey (Tabla 5), mostrando diferencias significativas $(p<0.05)$ en todas las variables analizadas, corroborando lo descrito anteriormente mediante la estadística descriptiva básica mostrada en la Tabla 3.

\section{DISCUSIÓN}

Se detectó variabilidad dentro de los ciclos, siendo mayor en los subciclos otoño-invierno y segundos cultivos, con una tendencia a disminuir su superficie, incluso en los últimos seis ciclos agrícolas no se tiene ningún registro de estos cultivos, debido a un incremento gradual de los cultivos perennes, ejerciendo presión sobre la disponibilidad del recurso hídrico, por el incremento porcentual, principalmente de alfalfa con respecto a la superficie total en el DR-
05 (CONAGUA 2018), lo que ocasionó una reducción en los otros cultivos. Esta tendencia de crecimiento ha convertido al DR-05 en una región especializada en este cultivo, también los cultivos de cebolla, chile, nogal y cacahuate, son cultivos en los que el DR-05 se ha vuelto referente y líder a nivel nacional (SEMARNAT 2017).

El análisis de correlación entre pares de variables demostró asociación lineal positiva, la cual fue estadísticamente significativa en la mayoría de los casos. El análisis de Componentes Principales indicó que los CP1 y CP2, explican el $81 \%$ de la variabilidad total del sistema, lo que es superior al $75 \%$ recomendado, y permitió identificar los cultivos importantes diferenciándolos de forma significativamente del resto en base a las variables de eficiencia de riego y eficiencia económica en los cuales se tiene una especialización productiva como el cultivo de chile, cebolla, alfalfa, nogal y cacahuate, que tienen rendimientos por arriba de la media nacional, 
Tabla 5. Prueba de comparación de medias* de Tukey $(p<0.05)$ de VALVOL, VALHA y VOLHA.

\begin{tabular}{|c|c|c|c|c|c|c|c|c|c|}
\hline \multirow{3}{*}{ Alfalfa } & \multicolumn{3}{|c|}{ VALVOL } & \multicolumn{3}{|c|}{ VALHA } & \multicolumn{3}{|c|}{ VOLHA } \\
\hline & \multicolumn{2}{|c|}{ Media } & \multirow{2}{*}{$\begin{array}{l}\text { E.E. } \\
351.09\end{array}$} & \multicolumn{2}{|c|}{ Media } & \multirow{2}{*}{$\begin{array}{l}\text { E.E. } \\
5541.51\end{array}$} & \multicolumn{2}{|c|}{ Media } & \multirow{2}{*}{$\begin{array}{l}\text { E.E. } \\
0.57\end{array}$} \\
\hline & $2463.17^{b c}$ & \pm & & $40319.83^{b}$ & \pm & & $16.49^{a}$ & \pm & \\
\hline Algodón & $4696.00^{b c}$ & \pm & 485.39 & $47379.33^{b}$ & \pm & 3464.61 & $10.28^{d}$ & \pm & 0.58 \\
\hline Cacahuate & $2867.50^{b c}$ & \pm & 262.90 & $31784.50^{b}$ & \pm & 1903.12 & $11.26^{c d}$ & \pm & 0.41 \\
\hline Cebolla & $14298.00^{a}$ & \pm & 2290.56 & $144959.17^{a}$ & \pm & 23672.21 & $10.05^{d}$ & \pm & 0.76 \\
\hline Chile & $9740.50^{a b}$ & \pm & 1495.39 & $150610.67^{a}$ & \pm & 22931.40 & $15.56^{a b}$ & \pm & 0.54 \\
\hline Maíz & $2060.67^{c}$ & \pm & 348.80 & $27151.67^{b}$ & \pm & 4082.63 & $13.85^{a b c}$ & \pm & 1.06 \\
\hline Nogal & $7278.83^{a b c}$ & \pm & 1082.13 & $101792.83^{a b}$ & \pm & 14842.52 & $14.16^{a b c}$ & \pm & 0.54 \\
\hline Sandia & $8963.67^{a b c}$ & \pm & 2003.91 & $106178.33^{a b}$ & \pm & 18317.98 & $12.44^{b c d}$ & \pm & 0.87 \\
\hline Varios & $12203.00^{a}$ & \pm & 3242.84 & $139000.00^{a}$ & \pm & 33836.37 & $12.46^{b c d}$ & \pm & 1.13 \\
\hline
\end{tabular}

lo que genera competencia interna entre productores, ocasionando que el precio promedio pagado este por debajo de la media nacional. La cebolla fue el cultivo con mejor eficiencia productiva y económica, en los últimos ciclos ha incrementado su rendimiento y redujo sus consumos de agua por unidad de superficie mostrando una mejora sustancial en la eficiencia física del uso del agua comparativamente con los valores reportados de la utilización de este recurso en México (SAGARPA 2018), de igual manera mostró valores muy superiores en lo relativo a producción por unidad física con respecto a lo manifestado por Rao et al. (2019) de $45.25 \mathrm{t} \mathrm{ha}^{-1}$, pero con menor eficiencia de productividad del agua en comparación con los $12.36 \mathrm{t} \mathrm{dam}^{-3}$, y en lo relativo a VALVOL este cultivo

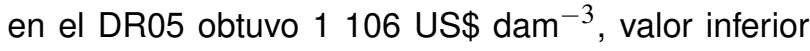
a lo expresado por estos autores en su trabajo de investigación realizado bajo condiciones controladas.

Mediante el análisis clúster se logró identificar y agrupar los cultivos en base a las diferentes eficiencias productivas y económicas en dos grupos, los de mayores eficiencias lo tuvieron la cebolla, alfalfa y nogal. El otro grupo conformado por los de menores eficiencias, menor ingreso, pero mayor seguridad en lo productivo y económico para los agricultores, se encuentran el cacahuate, algodón y los cultivos forrajeros como maíz, trigo y avena, con demanda interna en el distrito de riego. Al realizar la validación del modelo general, mediante MANOVA, en los grupos mediante contrastes y entre cultivos por comparación de medias de Tukey, resultó altamente significativo el modelo multivariado, encontrando diferencias alta- mente significativas entre los grupos y cultivos. Aun cuando se observaron mejorías en la eficiencia el uso del agua de riego, es necesario reducir más los valores en este apartado, mejorando la eficiencia de conducción y la eficiencia de aplicación, principalmente en el cultivo de alfalfa, ya que la superficie establecida utilizó más de la mitad del volumen total en el DR-05 en el ciclo 2017-2018, donde el 75\% de los productores utilizan aun riego tradicional (Villalobos et al. 2018).

\section{CONCLUSIONES}

Se detectaron dos tipos de alternativas productivas, en base al riesgo y tipo de productor. Por una parte, los cultivos de especialización productiva, de mayor eficiencia económica, alto ingreso, pero con mayor variabilidad en la demanda-precio y la relación beneficio-costo. Por otra parte, los cultivos de menor riesgo productivo y económico, pero con una relación beneficio-costo menor. La variabilidad en la información evidenció que no hay planeación ni programación de la producción, por lo que se utilizan más recursos de los necesarios. Se generaron indicadores de eficiencia productiva y económica, el análisis e interpretación ayuda a la toma de decisiones asertivas con criterios de sustentabilidad, aportando beneficios económicos, sociales y ambientales. Aun cuando ha habido un avance en la eficiencia con respecto al volumen utilizado, se debe mejorar este aspecto. Por lo que se recomienda trabajar en una planeación con criterios 
de sustentabilidad a largo plazo. Se requieren realizar estudios a mayor profundidad y obtener información confiable mediante muestreos aleatorios que permitan caracterizar con mayor certeza la problemática y alternativas que mejoren la productividad, y permitan mejorar el balance hídrico, incrementando la sustentabilidad del área con impacto sobre el sistema.

\section{LITERATURA CITADA}

Amaro-Rosales M, De Gortari-Rabiela R (2016) Innovación inclusiva en el sector agrícola mexicano: los productores de café en Veracruz. Economía Informa 400: 86-104.

Álvarez R, Massa F (2015) Determinación de tipologías de infecciones parasitarias intestinales, en escolares mediante, técnicas de clustering sobre datos binarios. IESTA, Instituto de Estadística de la Universidad de la República. Montevideo, Uruguay. 19p

Aravena C, Hofman A (2014) Crecimiento económico y productividad en América Latina. CEPAL, Naciones Unidas. Santiago de Chile, Chile. 41p.

Avilez JP, Escobar P, Von Fabeck G, Villagran K, García F, Matamoros R, García A (2010) Caracterización productiva de explotaciones lecheras empleando metodología de análisis multivariado. Revista Científica FCV-LUZ 20: $74-80$.

Brenes-Gámez A, de León-Mojarro B, Rendón-Pimentel L (2000) Sistema de control adaptable para la regulación de canales de riego. Ingeniería Hidráulica en México 15: 63-72.

CONAGUA (2014) Estadísticas Agrícolas de los Distritos de Riego Año Agrícola 2012-2013. Comisión Nacional del Agua. Tlalpan, México, D.F. https://www.gob.mx/conagua/documentos/estadisticas-agricolas-delos-distritos-de-riego. Fecha de consulta: febrero 12, 2019.

CONAGUA (2018) Estadísticas Agrícolas de los Distritos de Riego Año Agrícola 2016-2017. Comisión Nacional del Agua. Tlalpan, México, D.F. https://www.gob.mx/conagua/documentos/estadisticas-agricolas-delos-distritos-de-riego. Fecha de consulta: mayo 12, 2019.

Hernández-Sampieri R, Fernández C, Baptista P (2010) Metodología de la investigación. Quinta edición, Mc Graw-Hill. México. 613p.

Hofman A, Mas M, Aravena C, Guevara JFD (2017) Crecimiento económico y productividad en Latinoamérica. El proyecto LA-KLEMS. El Trimestre Económico 84: 259-306.

Olvera-Salgado MD, Ojeda W, Bahena G, Alpuche O (2014) Participación y apropiación de la modernización y tecnificación del riego en Chihuahua México. Revista Ingeniería Hidráulica y Ambiental 35: 47-61.

Ortega-Gaucin D, Mejía Sáenz R, Palacios Vélez E, Rendón Pimentel L, Exebio García A (2009). Modelo de optimización de recursos para un distrito de riego. Revista Terra Latinoamericana 27: 219-226.

Palacio-Velez E, Mejia-Saez E (2011) Uso del agua en el sector agrícola en México. En: Oswald SU (Coord.) Retos de la Investigación del agua en México. UNAM, Centro Regional de Investigación Multidisciplinaria. Cuernavaca, Morelos. pp: 179-192.

Rao KVR, Gangwar S, Aherwar P, Yadav, D (2019). Growth, yield, economics and water use efficiency of onion (Allium cepa L.) under different micro irrigation systems. Journal of Pharmacognosy and Phytochemistry 8: 3866-3869.

Ríos JL, Rios BE, Cantú JE, Rios HE, Armendáriz S, Chávez JE, Navarrete C, Castro R (2017) Análisis de la eficiencia física, económica y social del agua en espárrago (Asparagus officinalis L.) y uva (Vitis vinífera) 
de mesa del DR-037 Altar-Pitiquito, Caborca, Sonora, México 2014. Revista de la Facultad de Ciencias Agrarias UNCUYO 50: 101-122.

Salazar-Moreno R, Rojano-Aguilar A, López-Cruz IL (2014) La eficiencia en el uso del agua en la agricultura controlada. Revista Tecnología y Ciencias del Agua 5: 177-183.

Sánchez RA, Servín M, Gutiérrez H, Serna A (2017) Eficiencia en el uso del agua de variedades de alfalfa (Medicago sativa L.) con sistema de riego subsuperficial. Revista Mexicana de Ciencias Pecuarias 8: 429435.

SAGARPA (2018) Sistema de información agroalimentaria y pesquera. Secretaría de Agricultura Ganadería Pesca y Alimentación. http://www.siap.sagarpa.gob.mx/. Fecha de consulta: 30 de mayo 2019.

SEMARNAT (2017) Estadísticas agrícolas de los distritos de riego Año agrícola 2015-2016. Secretaria del medio ambiente y recursos naturales. Tlalpan, México. 405p.

Tapella E, Rodríguez-Bilella PD (2014) Sistematización de experiencias: una metodología para evaluar intervenciones de desarrollo. Revista de Evaluación de Programas y Políticas Públicas 3: 80-116.

Villalobos O, Sánchez E, Morales CR, Esparza ME, Santellano E (2018) Análisis de la eficiencia productiva del cultivo de alfalfa mediante regresión logística de datos categóricos en el Distrito de Riego 05-Delicias, Chihuahua, México. Nova Scientia 10: 352-368. 